\title{
Computer Simulation of Ion Beam Analysis: Possibilities and Limitations
}

\author{
M. Mayer ${ }^{\mathrm{a}}$ W. Eckstein ${ }^{\mathrm{a}}$ H. Langhuth ${ }^{\mathrm{a}}$ F. Schiettekatte ${ }^{\mathrm{b}}$ \\ U. von Toussaint ${ }^{\mathrm{a}}$ \\ ${ }^{a}$ Max-Planck-Institut für Plasmaphysik, EURATOM Association, Boltzmannstr. 2, \\ D-85748 Garching, Germany \\ ${ }^{\mathrm{b}}$ Département de Physique, Université de Montréal, Succursale Centre Ville, \\ Montréal (Québec), Canada H3T 1J4
}

\begin{abstract}
Quantitative application of ion beam analysis methods, such as Rutherford backscattering, elastic recoil detection analysis, and nuclear reaction analysis, requires the use of computer simulation codes. The different types of available codes are presented, and their advantages and weaknesses with respect to underlying physics and computing time requirements are discussed. Differences between different codes of the same type are smaller by about one order of magnitude than the uncertainty of basic input data, especially stopping power and cross section data. Even very complex sample structures with elemental concentration variations with depth or laterally varying structures can be simulated quantitatively. Laterally inhomogeneous samples generally result in an ambiguity with depth profiles. The optimization of ion beam analysis measurements is discussed, and available tools are presented.
\end{abstract}

Key words: Computer software, Data analysis, Simulation, Ion beam analysis

\section{Introduction}

Except for the simplest cases, the quantitative application of ion beam analysis (IBA) methods, such as Rutherford backscattering (RBS), elastic recoil detection analysis (ERDA) and non-resonant nuclear reaction analysis (NRA), requires the use of computer simulation codes for the analysis of measured particle energy spectra. Available simulation codes for this purpose were lastly reviewed about 5 years ago [1], with some updates in [2]. Some of the conclusions from this review were meanwhile implemented by a software intercomparison organized by the International Atomic Energy Agency (IAEA) [3,4], namely 
the validation of existing methods, estimation of errors in the information extracted by different programs, and quantification of differences produced by different algorithms and implementations. During the last five years a steady development of computer codes took place, while the increasing computer power (especially by the increasing ability for parallel computing) made new applications possible and allowed to drop some approximations which were necessary due to computing time limitations.

This article tries to review current developments and is organized as follows: Improvements and remaining problems of basic input data (stopping powers, straggling data, cross-sections) are discussed in section 2. The different types of available simulation codes are presented and discussed in section 3, the accuracy of codes is discussed in section 3.4. Analysis of complex samples (with emphasis on the analysis of of laterally inhomogeneous samples) is discussed in section 4. The optimization of IBA measurements is discussed in section 5 .

This paper limits itself to simulation codes for energy spectra of charged particles in random materials. Direct calculation codes without simulation, for example LORI [5], SQUEAKIE [6] or KONZERD [7], are not considered. Simulation of methods involving photons, such as PIXE [8] and PIGE, as well as channeling in single crystalline materials [9], are also not considered. It should be noted, however, that some of the simulation problems depicted in this paper, such as inaccuracies of stopping powers or ambiguities due to lateral sample inhomogeneities, also may affect these methods.

\section{Input data}

Quantitative application of IBA methods requires precise basic input data, especially stopping power data, straggling parameters, and cross-section data. As will be shown below, the precision of IBA methods is largely governed by the inaccuracy of these input data. This inaccuracy is also the main driver for the precision of simulation codes: It usually does not make sense to implement highly precise but slow algorithms, if the precision of input data is small.

\subsection{Stopping power data}

Although not the only available program for the calculation of stopping powers [10], the SRIM code [11,12] has become the de-facto standard for stopping power calculations. The SRIM module, firstly introduced with SRIM-2003, allows an easy integration of SRIM stopping power calculations into external programs [12] - one important reason for the popularity of SRIM stopping 
powers.

The last major changes to SRIM stopping powers were introduced in SRIM1995, SRIM-1998 and SRIM-2003. In 1995 a complete overhaul of the stopping of relativistic light ions with energies above $1 \mathrm{MeV} / \mathrm{amu}$ was made, while in 1998 the Barkas Effect and theoretical stopping of Li ions were improved. In 2003, new theoretical calculations for the stopping of heavy ions at lower energies were included. The accuracy of SRIM-2003 for H-ions is about $4.2 \%$, for He ions $4.1 \%$, for $\mathrm{Li}$ ions $5.1 \%$, and for heavier ions (from Be to U) 6.1\%, resulting in an overall accuracy of 4.8\% [12]. Compared to SRIM-1998 with an overall accuracy of $6.1 \%$ the accuracy of stopping powers increased, but only at a slow pace. Recent versions of SRIM (until SRIM-2010) did not change the SRIM-2003 stopping powers. It could be demonstrated that the SRIM-2003 stopping power for $1.5 \mathrm{MeV}^{4} \mathrm{He}$ in $\mathrm{Si}$ is accurate within $0.6 \%$ [4], but this high precision is a rare exception: The uncertainty of stopping powers is often the major source of uncertainty for the computer simulation of ion beam analysis spectra.

IBA simulation programs usually employ a linear addition of stopping powers (known as Bragg's rule) for determining the stopping power in compounds [13]. However, as is well known, Bragg's rule can be incorrect within up to $20 \%$ [12], especially in light target materials such as hydrocarbons. In heavy compounds (such as $\mathrm{BaCl}_{2}, \mathrm{Fe}_{2} \mathrm{O}_{3}, \mathrm{WO}_{3}, \mathrm{ZnO}, \ldots$ ) the deviation from Bragg's rule is typically below $2 \%$. A number of simulation programs allow to take deviations from Bragg's rule into account, either by using individual stopping power data for layer molecules or whole layers, or by employing a correction factor to the stopping power obtained from Bragg's rule. However, this possibility is only seldom applied by users. It's use is strongly encouraged.

\subsection{Straggling data}

Electronic energy loss is always associated with electronic energy-loss straggling [14]. State-of-the-art simulation programs take the binding of electrons to the atomic cores, charge state fluctuations of the projectile ion, and propagation of straggling in thick layers into account [15]. Energy-loss straggling is often assumed to have a Gaussian shape, but this is only approximately correct in a narrow range of energy losses. For very small energy losses, i.e. close to the surface, and for larger energy losses, i.,e. deeper in the sample, the particle energy distributions may deviate considerably from a Gaussian [14]. Recent developments of simulation programs take this non-Gaussian shape into account. In [16] a $\Gamma$-distribution is used to model the straggling distribution. Although this approach is problematic because it generally does not reproduce correctly the third moment of the distribution, it can result in an 
improved approximation in a number of cases. In [17] the skewness of the energy-loss straggling distribution is taken into account by its third moment. The straggling distribution is then approximated by a two-piece normal distribution, thus reproducing the mean value, width, and skewness of the energyloss straggling distribution. This approach can be generalized to take also the skewness of multiple scattering and geometrical straggling distributions into account [17].

\subsection{Cross-section data}

At high energies scattering and recoil cross-sections deviate from the Rutherford cross-section due to the influence of the nuclear force. A very simple fit formula allowing to estimate the maximum energy until which the Rutherford cross-section can be used was published in [18] and is widely used. However, the underlying concept of an actual Coulomb barrier was criticized to be based on irrelevant physics and its predictions to be misleading [19], so that the results from [18] should be used with great care. The most reliable method to determine if a cross-section is still Rutherford or not is therefore a look at assessed experimental or evaluated theoretical cross-section data.

As a result of a technical meeting and a coordinated research project (CRP) at the International Atomic Energy Agency (IAEA) a database (Ion Beam Analysis Nuclear Data Library (IBANDL) [20]) was created containing experimental cross-section data for non-Rutherford scattering, non-Rutherford recoil creation, and nuclear reactions [21-23] - first proposals for such a crosssection data base date back to the early 90's [24]. The current content of IBANDL (August 2010) are 1874 cross-section data files, i.e. almost all measured and published cross-section data since the 50's are available in IBANDL in numerical form. Many of the files have been assessed, i.e. at least they were compared to the original publication and, as far as possible, to other available experimental data. The data are in R33 file format.

In addition to this huge collection of experimental cross-section data, 27 evaluated non-Rutherford cross-sections are available and 5 evaluated nuclearreaction cross-sections $[22,23]$. These evaluated cross-sections are based on nuclear models and available for any scattering or reaction angle. It should be noted, however, that the accuracy of these cross-sections has never been evaluated and is therefore uncertain.

The availability of evaluated cross-section data for a number of important light elements is a major step forward for the computer simulation of IBA. The availability of backscattering cross-section data at any angle enables as new application the calculation of non-Rutherford recoil cross-sections for incident 
medium heavy ions, for example ${ }^{1} \mathrm{H}\left({ }^{16} \mathrm{O},{ }^{1} \mathrm{H}\right){ }^{16} \mathrm{O}$ through inverse kinematics. This enables the quantitative application of medium heavy ion ERDA (MIERDA) for simultaneous depth profiling of hydrogen isotopes and helium [25].

At low energies the scattering cross-section deviates from the Rutherford crosssection due to partial screening of the nuclear charge by the surrounding electrons. Several easy computable screening correction functions have been developed for IBA applications for the weakly screened region, i.e. if the deviation from the Rutherford cross-section is below about 20\%. Often applied screening corrections are from L'Ecuyer [26] and Andersen [27]. These screening corrections are especially used by analytical simulation codes presented in section 3.3 and in [1]. Different types of screened potentials were developed for lower energies and are used by full Monte Carlo codes, see section 3.1: SRIM uses the ZBL or Universal potential [28], for TRIM.SP the Kr-C potential [29] is often used. Different screened cross-sections are compared in Fig. 1 for ${ }^{4} \mathrm{He}$ backscattered from Au at $165^{\circ}$. The Universal cross-section was calculated in [30] using Gauss-Mehler integration with 10000 points, thus providing very high accuracy. The tabulated data points are interpolated in Fig. 1 using quadratic interpolation between the logarithmic energy values. At $1 \mathrm{MeV}$ the Universal cross-section is about 1.5\% higher than Andersen and L'Ecuyer, at $500 \mathrm{keV}$ this difference increases to about $2.2 \%$. The difference between different screening corrections generally increases with decreasing scattering angle and decreasing energy. For high-Z elements and at typical energies used for IBA the uncertainty due to different screening corrections is therefore in the range of $1-2 \%$. This has to be kept in mind when comparing results from different types of codes, because full Monte Carlo codes traditionally use different screening corrections than analytical codes.

TRIM-like full Monte-Carlo codes usually don't compute the scattering integral accurately, but use the faster MAGIC approximation [31]. The accuracy of MAGIC for the calculation of the scattering angle is 1-2\% [30], resulting in a comparable inaccuracy for the scattering cross-section. While this is less problematic at low energies, where a large number of scattering events occur, it may introduce an additional error at typical energies used for IBA, where one scattering event may be dominant.

\section{Types of simulation codes}

Different types of simulation codes have been developed during the last three decades, based on different approximations to the underlying physics of ionsolid interactions. The codes can be roughly subdivided into three categories: Full Monte-Carlo, Monte-Carlo with weight function, and analytical code. Each of these types of codes has its strengths and weaknesses with respect to 
accuracy, computing time requirement, and usability.

\subsection{Full Monte-Carlo simulation}

In a full Monte-Carlo (MC) simulation the incident ion is tracked from the target surface until it finally comes to rest in the material (or its initial energy has decreased below some pre-set value) [31,32]. Typical representatives of this type of code are the popular SRIM-code [11,12] or SDTrimSP [33]. The codes described in section 3.2 (MC with weight function) can be often also used for full MC simulations. An amorphous material is assumed with a random free path length between subsequent collisions. The crystalline structure of the material can be taken into account in order to calculate channeling effects $[9,34,35]$. All scattering events including events with small deflection angles are taken into account, thus resulting in a very realistic simulation of multiple and plural scattering events. For the calculation of a scattering event the binary collision approximation is used. This approximation is very reasonable at typical IBA energies. For improved accuracy the influence of additional neighbors can be taken into account approximately. Most full MC codes were initially developed for the keV energy range, and the effects of electronic energy loss straggling and detector resolution are either oversimplified or not implemented. Non-Rutherford scattering cross-sections are not implemented, and (depending on selected screening function) the scattering cross-section may slightly deviate from other codes, see section 2.3. The codes presented in section 3.2 were developed for typical IBA energies and therefore suffer less from these limitations, when used for a full MC simulation.

Full MC simulation offers the highest precision in the simulation of IBA spectra, taking all relevant physical processes into account. Moreover, a full MC simulation code is very lean: The core requires only a few hundred lines of code. The identical code can be used from the several $10 \mathrm{eV}$ to the several $\mathrm{MeV}$ energy range, thus allowing easy testing and debugging of the code. The main problem of full MC codes are the much too long computing times: For solid angles of about $1 \mathrm{msr}$, as are typically used in IBA, and medium heavy targets, the backscattering yield is of the order of only $10^{-9}$, so that of the order of $10^{12}$ incident particles have to be calculated: This takes several years on current computer hardware. However, these codes can be easily parallelized and offer an almost linear speed-up with the number of processors. A speedup by a factor of 500 when running the code on 512 processors was already demonstrated for SDTrimSP [33], and comparable speed-ups on even larger clusters can be expected. Although at present and in the near future full MC simulation is not applicable for everyday work, it can be used for fundamental investigations, especially on the influence of multiple and plural scattering, and for testing and benchmarking other types of code. 


\subsection{Monte-Carlo with weight function}

Although the most accurate method, a full MC simulation requires too long computing times for practical applications, see section 3.1. The computing time was decreased by several orders of magnitude by a number of improvements:

(1) The target is kept as thin as possible, and only ions heading towards the detector are followed [36,37].

(2) Scattered/recoiled atoms are pitched only in detector direction, crosssections instead of counts are accumulated, and a virtual detector 100 times larger than the real one is used $[38,39]$.

(3) Computational improvements: The MAGIC procedure for the calculation of the scattering integral is replaced by matrix interpolation [40].

(4) Transcendent functions are avoided as far as possible and replaced by interpolations of pre-calculated values [41].

These improvements reduce the computing time for $\mathrm{MC}$ simulations from several years to a few minutes. Two different codes are publicly available: MCERD $[38,39]$ and CORTEO [41]. The approximations outlined above can be switched off, in which case these codes run a full MC simulation (with increased computing time). Both codes were initially developed for MeV IBA application, so their handling of MeV-specific effects, such as energy-loss straggling, is more accurate than by native full MC codes.

However, besides their dramatic decrease of computing time, the improvements sketched above introduce also some problems: The code gets more complex and is consequently more prone to bugs. Ions starting initially in directions away from the detector are not calculated, thus neglecting some plural scattering background. Backscattering simulations require the introduction of a minimum scattering angle, which is arbitrary and not physical this problem does not occur for recoils. The accumulation of cross-section instead of counts results in an awkward non-Poisson statistics and may contain visible spikes. The virtual detector requires a kinematic correction in order to avoid too large geometrical straggling effects. This kinematic correction is only approximate.

These types of code are more difficult to use than full MC simulation codes. CORTEO [41] has introduced an easy-to-use graphical user interface, thus allowing the use of this type of codes even for non-experts. 


\subsection{Analytical codes}

At sufficiently high energies and/or sufficiently light target materials ion trajectories can be approximated by straight lines for in- and outgoing particles with one scattering, recoil or reaction event combining them. The energy spectrum is then obtained as the solution of a number of coupled differential equations, which can be solved numerically. A number of codes have been developed for this purpose, an overview can be found in [1]. According to citation frequency the most popular are SIMNRA [42,43], RUMP $[44,45]$ and NDF [46], but other codes are also used. These codes are faster by several orders of magnitude, as compared to all types of MC codes. This allows the use of improved fitting algorithms for automatic or semi-automatic spectrum fitting [46] and the analysis of large numbers of spectra. Many of these codes have an easy-to-use graphical user interface and a data base of cross-sections for non-Rutherford scattering and nuclear reactions.

For not too large energy losses, multiple small-angle scattering can be treated as an additional energy broadening contribution, which can be calculated using an analytical theory of multiple scattering [15,47-49]. Several analytical simulation programs can include multiple scattering effects in this approximation, see [1] for details. This analytical theory was compared to MC simulation calculations and showed very good agreement [50] for small and medium energy losses. At oblique incidence the multiple scattering energy distributions get skewed, which can be additionally taken into account [17]. But at large energy losses the analytical theory of multiple scattering gets more and more inaccurate [50]. Consequently, with these types of codes the low energy part of IBA energy spectra can be simulated only, if multiple scattering does not play a role: This is usually only the case for very light target materials.

In addition to multiple small-angle scattering, trajectories with two or more large angle deflections may occur. This is often called plural scattering. Plural scattering trajectories result in additional spectrum intensity in areas of the spectrum where no intensity is expected in the single scattering approximation, for example below the low-energy edge of heavy element layers [51-54]. The effects of plural scattering cannot be calculated analytically. The dual scattering approximation [54] takes two large angle deflections into account, which, in many cases, already yields a reasonable approximation to the plural scattering background. This approximation typically somewhat underestimates the plural scattering background due to the neglect of trajectories with more than two large angle deflections [54]. Although it increases the computing time considerably, it is usually still fast enough even for daily work and especially important for thicker layers of heavy elements and at lower energies.

Analytical codes are the most widely used codes for IBA spectrum simulation 
and evaluation. A number of codes are publicly available, differing widely not only in the implemented physics and input data bases for stopping powers and cross-sections, but also in their user interface, extensibility, and automatic fitting capabilities. Many of the codes are being developed since many years, resulting in a very mature status without obvious bugs. Precise IBA analysis of samples containing heavy elements usually requires the use of codes with multiple and plural scattering simulation capabilities.

\subsection{Code accuracy}

The accuracy of 6 analytical and one MC code was determined in a software intercomparison for typical IBA problems using identical input data $[3,4]$. The differences between different codes were only about $0.1 \%$. This is much lower than the uncertainty of input data, especially of stopping powers and nonRutherford cross-sections. This small difference between different codes is not too astonishing, because code authors usually implement algorithms which are more accurate than input data by one to two orders of magnitude: A higher accuracy does not make sense and usually slows down the code, while a smaller accuracy results in visible deviations to measured spectra. The analytical multiple scattering theory from [15,47-49], which is implemented in a number of of analytical codes [1], was compared to Monte-Carlo calculations in binary collision approximation and molecular dynamics calculations in [50] and showed good agreement if the energy losses were not too large, see also section 3.3. The accuracy in the evaluation of IBA data is therefore dominated by the inaccuracies of basic input data and not by inaccuracies of algorithms implemented in simulation codes. Nevertheless, despite this overall result it should be noted that some codes showed larger deviations or were even incorrect in special cases, for example for ERDA [3].

\section{Complex samples}

\subsection{Laterally homogeneous samples}

All simulation codes are able to handle layered sample structures in slab geometry: The sample is assumed to consist of stacked layers, where each layer extends indefinitely parallel to the surface. Each layer has a homogeneous composition and a thickness. State-of-the art simulation codes either don't limit the number of layers at all, or they allow so many layers that the upper limit is not a practical limitation. Simulations with dozens of layers (for example stacked Co/Re layers) have been successfully performed [3]. 
Continuous concentration depth profiles, such as Gaussian shaped implantation profiles or diffusion profiles of individual elements, can be approximated by a larger number of thin layers. The element concentrations in each layer are constant, but vary from layer to layer. Some programs already have the build-in ability to generate special types of depth profiles (usually Gaussian or diffusion type profiles), while some other programs are extensible and external target editors have been written $[55,56]$, thus allowing to create even very complicated target structures. The approximation of continuous depth profiles by thin layers with constant concentrations is mathematically not fully correct, because the continuous change of the stopping power by a continuous concentration profile is not taken fully correctly into account: A number of mathematical methods exist, that could provide higher accuracy. However, the approximation of continuous profiles by layer slabs keeps both the simulation code and the user interface consistent and provides sufficient accuracy, if the layer thicknesses are small enough.

\subsection{Laterally inhomogeneous samples}

Practically, laterally homogeneous layered sample structures as described in section 4.1 are only available from semiconductor industry, which is one of the main fields of application for IBA. In all other cases samples usually have laterally inhomogeneous layer structures. This may be different types of layer or substrate roughness [57-59], island growth [60], incomplete coverage of the surface due to inhomogeneous layer deposition or erosion [61,62], randomly distributed microstructures [55] or inclusions [63], or regular gratings [64] or meshes, as are used for example for collecting dust samples from air.

A rough layer is characterized by a distribution of layer thicknesses. If the layer roughness is small compared to the mean layer thickness, then the influence of this roughness on the energy spectrum of backscattered particles can be treated as an additional Gaussian energy broadening contribution [59]. For larger roughnesses this contribution gets non-Gaussian and can be calculated as a linear superposition of energy spectra calculated for smooth layers [58]. The statistical weight of each sub-spectrum is determined from the probability distribution function of layer thicknesses. This ansatz neglects correlations between incident and exit beams (for example incidence through a valley and exit through a hilltop of the rough surface) and is therefore only valid if the lateral spacing of the roughness is larger than its vertical extension and for backscattering at angles close to $180^{\circ}$. Correlation effects can be taken into account analytically [65] or by Monte-Carlo simulations [66], but require additional knowledge about the roughness parameters. These can be obtained for example from atomic force microscopy (AFM) measurements. As probability distribution function for the layer thickness distribution the $\Gamma$-distribution has 
been proposed [67] and has proven to be a very successful approximation for rough layers in many cases $[67,68]$.

Substrate roughness can be taken into account using a similar ansatz with a distribution of local tilt angles, resulting in a distribution of incident and exit angles [67]. As in the case of layer roughness, correlation effects between incident and exit means are neglected. A Lorentz distribution [67] or a Gaussian distribution of local tilt angles have been used.

RBS spectra from rough Mo/W layers on top of $\mathrm{W}$ are shown in Fig. 2. Both examples require surface roughness, multiple and dual scattering for a successful simulation of the experimental spectra. Despite the visible layer roughness the spectrum from the left sample can be simulated accurately, except channel numbers below about 200: At these large energy losses the analytical multiple scattering model fails, see section 3.3. Plural scattering gives an important contribution at lower energies. The simulation of the spectrum from the right sample requires additionally a Lorentzian substrate roughness of $60^{\circ} \mathrm{FWHM}$ : This value for the roughness is based on profiler measurements of the CFC substrate. The simulation reproduces the main features of this spectrum and allows to extract the mean thicknesses of the Mo and W layers. However, some details of the experimental spectrum are not well reproduced, so that this very complex sample marks the current possibilities of the simulation of inhomogeneous, rough samples. A simple simulation assuming smooth layers and without plural scattering does not reproduce the experimental spectra.

\subsection{Ambiguity between lateral inhomogeneities and depth profiles}

The method of linear superposition of sub-spectra, as outlined in section 4.2, can be used not only for rough samples, but for arbitrary lateral structures $[60,55]$. As in the case of surface roughness, correlations between incident and exit beams are neglected, so that this method is only valid if the lateral extension of surface structures is much larger than their thickness.

Laterally inhomogeneous samples have to be analyzed with care due to a general ambiguity between concentration variations with depth, i.e. depth profiles, and thickness variations. This is shown in Fig. 3. The measured RBS spectrum can be described by two very different sample models: Model 1 assumes a laterally homogeneous sample structure with depth-dependent concentrations of Si and Ta. This model provides a good fit to the measured spectrum and requires the assumption of mixed SiTa layers at the top and bottom interfaces of the Ta layer. Model 2 assumes a lateral variation of the top $\mathrm{Si}$ layer thickness with two different thicknesses. The real sample cross-section is shown in Fig. 3 bottom. The top Si layer is a grating with two different thick- 
nesses, which can be extracted from model 2. The spectra calculated with the two different models are practically indistinguishable, although only model 2 reflects the real sample structure. This ambiguity between depth profile and lateral thickness variation is a very general feature of ion beam analysis, and practically each spectrum can be fitted either with a laterally homogeneous depth profile of elements or with a lateral thickness variation of layers. This ambiguity can easily result in incorrect conclusions, and is generally difficult to solve with ion beam analysis methods alone. The deduction of a depth profile and of conclusions based on it therefore always requires a proof, that the sample is laterally homogeneous. This is best done with laterally resolving non-IBA methods, such as scanning electron microscopy (SEM), focused ion beam cross sectioning (FIB), profilometry, atomic force microscopy (AFM) or scanning tunneling microscopy (STM). IBA measurements at a number of different geometries may also help to detect ambiguities [69]. It should be noted, though, that both models shown in Fig. 3 yield identical total amounts (in atoms $/ \mathrm{cm}^{2}$ ) of $\mathrm{Ta}$ and $\mathrm{Si}$ within the error bars of the measurement, i.e. the total amount of elements can be extracted robustly even in the case of ambiguities.

\section{$5 \quad$ Experimental design and planning}

All simulation codes can be used to design experiments by performing simulations to test different experimental parameters, such as ion energies, geometries, beam fluences etc. for an assumed sample structure. This allows to determine if the signals from different elements overlap, if the sensitivity of a setup is large enough to detect a specific signal, and so on. These test simulations are much faster and cheaper than a real measurement. However, in order to extract the maximum information available through IBA methods, the experimental parameters (such as incident ion species, incident energy, incident and exit angles) have to be optimized. Often the best achievable depth resolution in a specific depth is desired, although other optimization criteria (such as maximum sensitivity in a specific depth or maximum analyzable depth) may exist. These optimized parameters are depth and element specific.

The DEPTH [15] and RESOLNRA [70] codes calculate the depth resolution taking detector resolution, energy-loss straggling, geometrical straggling and multiple scattering energy spread into account. They help to find optimum incident energies and incident angles. The optimum depth resolution, especially close to the surface, often requires the use of very grazing incidence or exit angles of $80^{\circ}$ and more [70]. This is an experimental challenge.

A given combination of incident energy/incident angle usually provides an optimum depth resolution only in one specific depth for one specific element. In 
order to achieve an optimum depth resolution throughout a larger depth range or for multiple elements requires measurements at different energies or geometries [71,3]. Depth profiling of deuterium with a depth resolution of $1-2 \mu \mathrm{m}$ until depths of $40 \mu \mathrm{m}$ was demonstrated in amorphous deuterated hydrocarbon layers using the $\mathrm{D}\left({ }^{3} \mathrm{He}, \mathrm{p}\right) \alpha$ nuclear reaction at different incident energies [72]. Planning of such multiple energies measurements requires a thorough calculation of the achievable depth resolution, taking all relevant energy spread contributions into account. Multiple energies, geometries or methods may be also necessary to solve ambiguities of depth profiles of multiple elements [73].

The described optimization of measurements requires prior knowledge about the sample structure. If additional prior information about the shape of the depth profiles exists (e.g. an exponential decaying concentration profile), then this prior knowledge can be taken into account using Bayesian inference in order to minimize the number of necessary measurements [74].

\section{Conclusions}

Except for the simplest cases, the quantitative application of IBA methods requires the use of computer simulation codes for the analysis of measured particle energy spectra. Simulation codes for IBA are well developed. Three different types of codes have been developed for the simulation of IBA spectra: Full Monte Carlo simulation, Monte Carlo simulation with weight function, and analytical codes. Each type of code has advantages and weaknesses with respect to accuracy, completeness of basic physical processes, computing time requirement, and code complexity. The inaccuracies of basic input parameters, namely stopping powers, non-Rutherford cross-sections at high energies, and screening corrections at low energies, are larger by about one order of magnitude than differences between different codes. The accuracy of simulation calculations is therefore determined by the accuracy of these basic input data, and not by the codes.

Complex sample structures with both laterally homogeneous and inhomogeneous structures (surface roughness, lateral layer thickness variations) can be simulated, and quantitative information can be extracted. However, laterally inhomogeneous samples have to be analyzed with care due to the ambiguity between concentration variations with depth, i.e. depth profiles, and lateral layer thickness variations. IBA measurements allow a reliable extraction of depth profiles only for laterally homogeneous samples.

Optimization of the depth resolution is usually only possible for one specific element and depth. The achievement of an optimum depth resolution throughout a larger depth range or for multiple elements requires measurements at 
different energies or geometries. This requires a thorough experimental design and planning.

\section{Acknowledgements}

The Mo/W samples were manufactured by C. Ruset from the National Institute for Laser, Plasma and Radiation Physics in Bucharest, Romania. Metallographic sample cross sections and electron microscopy by G. Matern are gratefully acknowledged.

\section{References}

[1] E. Rauhala, N.P. Barradas, S. Fazinic, M. Mayer, E. Szilágyi, and M. Thompson. Nucl. Instr. Meth. B 244 (2006) 436.

[2] N.P. Barradas and E. Rauhala. Data analysis software for ion beam analysis. In Wang and Nastasi [75], ch. 14.

[3] N.P. Barradas, K. Arstila, G. Battistig, M. Bianconi, N. Dytlewski, C. Jeynes, E. Kótai, G. Lulli, M. Mayer, E. Rauhala, E. Szilágyi, and M. Thompson. Nucl. Instr. Meth. B 262 (2007) 281.

[4] N.P. Barradas, K. Arstila, G. Battistig, M. Bianconi, N. Dytlewski, C. Jeynes, E. Kótai, G. Lulli, M. Mayer, E. Rauhala, E. Szilágyi, and M. Thompson. Nucl. Instr. Meth. B 266 (2008) 1338.

[5] B.M.U. Scherzer, H.L. Bay, R. Behrisch, P. Børgesen, and J. Roth. Nucl. Instr. Meth. 157 (1978) 75.

[6] P. Børgesen, R. Behrisch, and B.M.U. Scherzer. Appl. Phys. A 111 (1982) 717.

[7] A. Bergmaier, G. Dollinger, and C.M. Frey. Nucl. Instr. Meth. B 99 (1995) 488.

[8] J.L. Campbell. Particle-induced X-ray emission:PIXE. In Wang and Nastasi [75], ch. 11.

[9] M.L. Swanson and L. Shao. Ion channeling. In Wang and Nastasi [75], ch. 12.

[10] H. Paul. http://www.exphys.uni-linz.ac.at/stopping/.

[11] J.F. Ziegler. SRIM: The stopping and range of ions in matter. http://www.srim.org/.

[12] J.F. Ziegler. Nucl. Instr. Meth. B 219-220 (2004) 1027.

[13] W.H. Bragg and R. Kleeman. Philos. Mag. 10 (1905) 318. 
[14] P. Sigmund. Particle Penetration and Radiation Effects, vol. 151 of Springer Series in Solid State Sciences. Springer, Berlin, Heidelberg, 2006.

[15] E. Szilágyi, F. Pászti, and G. Amsel. Nucl. Instr. Meth. B 100 (1995) 103.

[16] N.P. Barradas, R.P. Pezzi, and I.J.R. Baumvol. Nucl. Instr. Meth. B 261 (2007) 422.

[17] M. Mayer, K. Arstila, and U. von Toussaint. Nucl. Instr. Meth. B 268 (2010) 1744 .

[18] M. Bozoian. Actual coulomb barriers. In Handbook of Modern Ion Beam Materials Analysis, J. Tesmer and M. Nastasi, Eds., first ed. Materials Research Society, Pittsburgh, Pennsylvania, 1995.

[19] A.F. Gurbich. Nucl. Instr. Meth. B 217 (2004) 183.

[20] Ion Beam Analysis Nuclear Data Library (IBANDL). http://wwwnds.iaea.org/ibandl/.

[21] A. Gurbich, I. Bogdanovic-Radovic, M. Chiari, C. Jeynes, M. Kokkoris, A.R. Ramos, M. Mayer, E. Rauhala, O. Schwerer, Shi Liqun, and I. Vickridge. Nucl. Instr. Meth. B 266 (2008) 1198.

[22] A. Gurbich. Nucl. Instr. Meth. B 268 (2010) 1703.

[23] D. Abriola, A.F. Gurbich, N.P. Barradas, I. Bogdanovic-Radovic, M. Chiari, C. Jeynes, M. Kokkoris, M. Mayer, A.R. Ramos, L. Shi, and I. Vickridge. Nucl. Instr. Meth. B (2011) . these proceedings.

[24] I. Vickridge. Nucl. Instr. Meth. B 66 (1992) 303.

[25] E. Markina, M. Mayer, and H.T. Lee. Nucl. Instr. Meth. B (2011) . these proceedings.

[26] J. L'Ecuyer, J.A. Davies, and N. Matsunami. Nucl. Instr. Meth. 160 (1979) 337.

[27] H.H. Andersen, F. Besenbacher, P. Loftager, and W. Möller. Phys. Rev. A21, 6 (1980) 1891.

[28] J.F. Ziegler, J.P. Biersack, and U. Littmark. The Stopping and Range of Ions in Solids, vol. 1 of The Stopping and Ranges of Ions in Matter. Pergamon Press, New York, 1985.

[29] W.D. Wilson, L.G. Haggmark, and J.P. Biersack. Phys. Rev. B15 (1977) 2458.

[30] H.G. Schlager and W. Eckstein. The scattering integrals - integration and accuracy. Tech. Rep. IPP 9/69, Max-Planck-Institut für Plasmaphysik, Garching, 1991.

[31] J.P. Biersack and L.G. Haggmark. Nucl. Instr. Meth. 174 (1980) 257.

[32] J.P. Biersack and W. Eckstein. Appl. Phys. A34 (1984) 73. 
[33] W. Eckstein, R. Dohmen, A. Mutzke, and R. Schneider. SDTrimSP: A MonteCarlo code for calculating collision phenomena in randomized targets. Tech. Rep. IPP 12/3, Max-Planck-Institut für Plasmaphysik, Garching, 2007.

[34] L. Nowicki, A. Turos, R. Ratajczak, A. Stonert, and F. Garrido. Nucl. Instr. Meth. B 240 (2005) 277.

[35] A. Turos, L. Nowicki, A. Stonert, K. Pagowska, J. Jagielski, and A. Muecklich. Nucl. Instr. Meth. B 268 (2010) 1718.

[36] R. D. Franich, P. N. Johnston, I. F. Bubb, N. Dytlewski, and D. D. Cohen. Nucl. Instr. Meth. B 190 (2002) 252.

[37] R. D. Franich, P. N. Johnston, and I. F. Bubb. Nucl. Instr. Meth. B 219-220 (2004) 87.

[38] K. Arstila, T. Sajavaara, and J. Keinonen. Nucl. Instr. Meth. B 174 (2001) 163.

[39] K. Arstila, J.A. Knapp, K. Nordlund, and B.L. Doyle. Nucl. Instr. Meth. B 219-220 (2004) 1058.

[40] B. Yuan, P.C. Yu, and S.M. Tang. Nucl. Instr. Meth. B 83 (1993) 413.

[41] François Schiettekatte. Nucl. Instr. Meth. B 266 (2008) 1880.

[42] M. Mayer. SIMNRA user's guide. Tech. Rep. IPP 9/113, Max-Planck-Institut für Plasmaphysik, Garching, 1997.

[43] M. Mayer. SIMNRA, a simulation program for the analysis of NRA, RBS and ERDA. In Proceedings of the 15th International Conference on the Application of Accelerators in Research and Industry (Woodbury, New York, 1999), J. L. Duggan and I. Morgan, Eds., vol. 475 of AIP Conference Proceedings, American Institute of Physics, p. 541.

[44] R. Doolittle. Nucl. Instr. Meth. B 9 (1985) 344.

[45] R. Doolittle. Nucl. Instr. Meth. B 15 (1986) 227.

[46] C. Jeynes, N.P. Barradas, P.K. Marriott, G. Boudreault, M. Jenkin, E. Wendler, and R.P. Webb. J. Phys. D: Appl. Phys. 36 (2003) R97.

[47] E. Szilágyi. Nucl. Instr. Meth. B 161-163 (2000) 37.

[48] G. Amsel, G. Battistig, and A. L'Hoir. Nucl. Instr. Meth. B 201 (2003) 325.

[49] G. Amsel, G. Battistig, and A. L'Hoir. Nucl. Instr. Meth. B 219-220 (2004) 1037.

[50] M. Mayer, K. Arstila, K. Nordlund, E. Edelmann, and J. Keinonen. Nucl. Instr. Meth. B 249 (2006) 823.

[51] A. Weber, H. Mommsen, W. Sarter, and A. Weller. Nucl. Instr. Meth. 198 (1982) 527.

[52] A. Weber and H. Mommsen. Nucl. Instr. Meth. 204 (1983) 559. 
[53] E. Steinbauer, P. Bauer, and J.P. Biersack. Nucl. Instr. Meth. B 45 (1990) 171.

[54] W. Eckstein and M. Mayer. Nucl. Instr. Meth. B 153 (1999) 337.

[55] M. J. F. Healy, M. Torres, and J. D. Painter. Nucl. Instr. Meth. B 249 (2006) 789.

[56] J.L. Colaux. Simtarget. http://webapps.fundp.ac.be/physique/simtarget/.

[57] N.P. Barradas. J. Phys. D: Appl. Phys. 34 (2001) 2109.

[58] M. Mayer. Nucl. Instr. Meth. B 194 (2002) 177.

[59] N.P. Barradas, E. Alves, S. Pereira, V.V. Shvartsman, A.L. Kholkin, E. Pereira, K.P. O'Donnell, C. Liu, C.J. Deatcher, I.M. Watson, and M. Mayer. Nucl. Instr. Meth. B 217 (2004) 479.

[60] M. Mayer, R. Fischer, S. Lindig, U. von Toussaint, R.W. Stark, and V. Dose. Nucl. Instr. Meth. B 228 (2005) 349.

[61] M. Mayer, J. Likonen, J.P. Coad, H. Maier, M. Balden, S. Lindig, E. VainonenAhlgren, V. Philipps, and JET-EFDA Contributors. J. Nucl. Mater. 363-365 (2007) 101.

[62] M. Mayer, V. Rohde, G. Ramos, E. Vainonen-Ahlgren, J. Likonen, J.L. Chen, and ASDEX Upgrade team. Physica Scripta T128 (2007) 106.

[63] J.P. Stoquert and T. Szörényi. Phys. Rev. B66 (2002) 144108.

[64] I. Bizyukov, A. Mutzke, R. Schneider, and J. Davis. Nucl. Instr. Meth. B 268 (2010) 2631.

[65] S.L. Molodtsov, A.F. Gurbich, and C. Jeynes. J. Phys. D 41 (2008) 205303.

[66] I.M. Yesil, W. Assmann, H. Huber, and K.E.G. Löbner. Nucl. Instr. Meth. B 136-138 (1998) 623.

[67] M. Mayer, J. Roth, and K. Ertl. Nucl. Instr. Meth. B 190 (2002) 405.

[68] P. Rao, S. Kumar, G.L.N. Reddy, S. Veena, S. Kalavathi, J.V. Ramana, and V.S.Raju. Nucl. Instr. Meth. B 268 (2010) 3395.

[69] J.R. Bird, P. Duerden, D.D. Cohen, G.B. Smith, and P. Hillery. Nucl. Instr. Meth. 218 (1983) 53.

[70] M. Mayer. Nucl. Instr. Meth. B 266 (2008) 1852.

[71] N.P. Barradas. Nucl. Instr. Meth. B 190 (2004) 247.

[72] M. Mayer, E.Gauthier, K. Sugiyama, and U. von Toussaint. Nucl. Instr. Meth. B 267 (2009) 506.

[73] C. Jeynes and N.P. Barradas. Pitfalls in ion beam analysis. In Wang and Nastasi [75], ch. 15. 
[74] U. von Toussaint, T. Schwarz-Selinger, M. Mayer, and S. Gori. Nucl. Instr. Meth. B 268 (2010) 2115.

[75] Y. Wang and M. Nastasi, Eds. Handbook of Modern Ion Beam Materials Analysis, second ed. Materials Research Society, Warrendale, Pennsylvania, 2009 . 


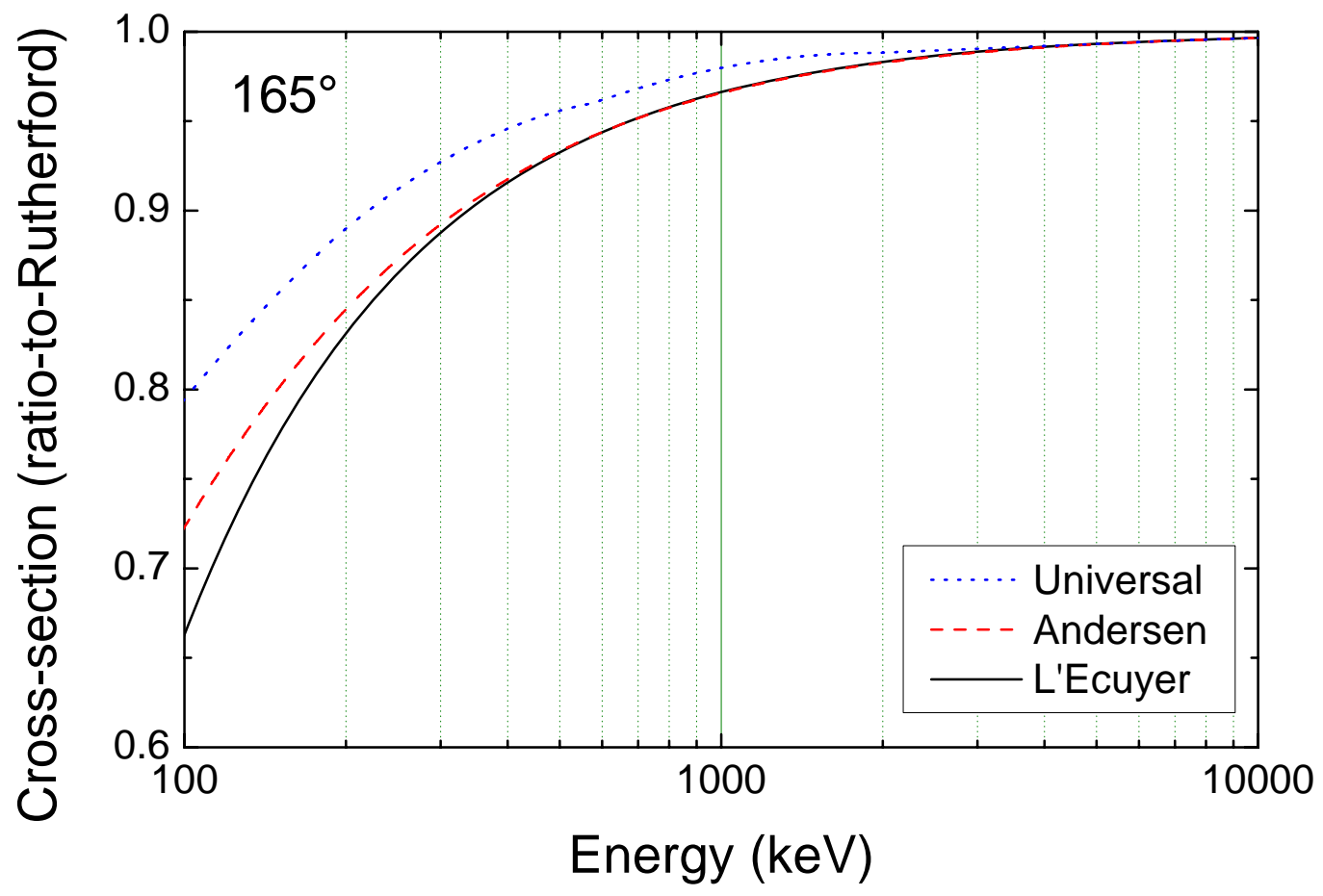

Fig. 1. Cross-section for backscattering of ${ }^{4} \mathrm{He}$ from $\mathrm{Au}$ at $165^{\circ}$. Universal: Universal potential from [28]; Andersen: Screening function from [27]; L'Ecuyer: Screening function from $[26]$. 

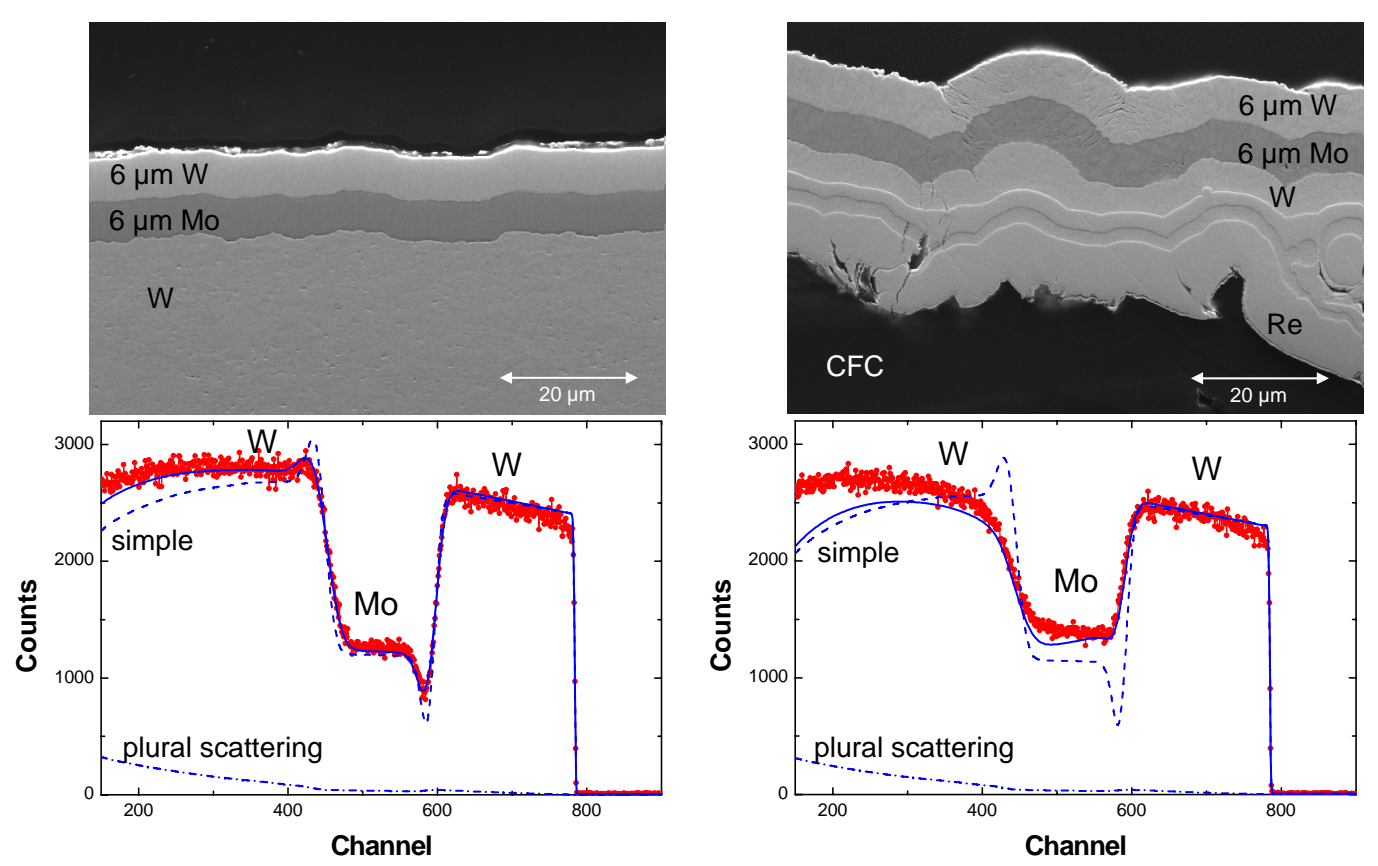

Fig. 2. Top left: Metallographic cross-section of $6 \mu \mathrm{m}$ Mo and W layers on bulk W. Top right: Metallographic cross-section of carbon-fibre composite (CFC) material, coated with a $14 \mu \mathrm{m}$ Re and W layer, $6 \mu \mathrm{m}$ Mo, and $6 \mu \mathrm{m}$ W. Bottom: Experimental and simulated RBS spectra from the samples, measured with $4 \mathrm{MeV}$ incident protons, backscattering angle $165^{\circ}$, normal incidence. Dashed line: Simple simulation, assuming homogeneous layers and no plural scattering. Dash-dotted line: Plural scattering contribution. Solid line: Simulation including substrate roughness (in case of CFC), layer roughness, multiple and plural scattering. Simulations by SIMNRA [42]. 


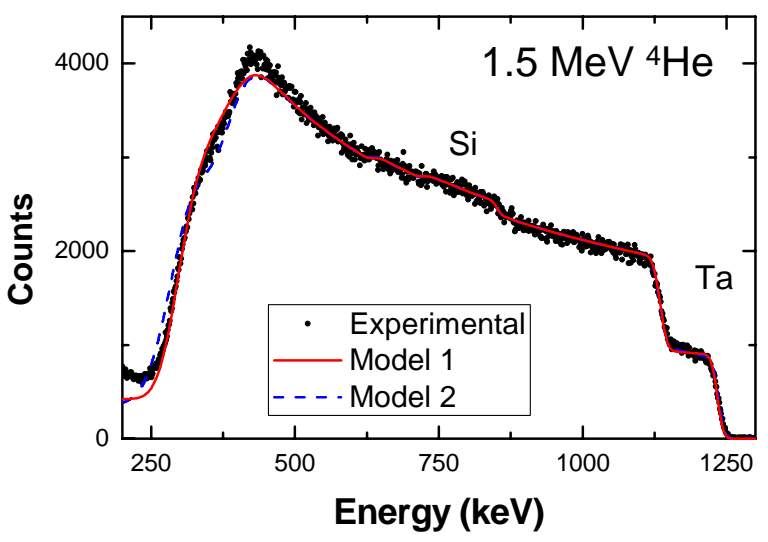

Model 1

Model 2

\begin{tabular}{lr}
$\mathrm{Si}$ & 1300 \\
$\mathrm{Si}_{0.7} \mathrm{Ta}_{0.3}$ & 670 \\
$\mathrm{Ta}$ & 3250 \\
$\mathrm{Si}_{0.5} \mathrm{Ta}_{0.5}$ & 550 \\
\multicolumn{3}{c}{$\mathrm{Si}$} &
\end{tabular}
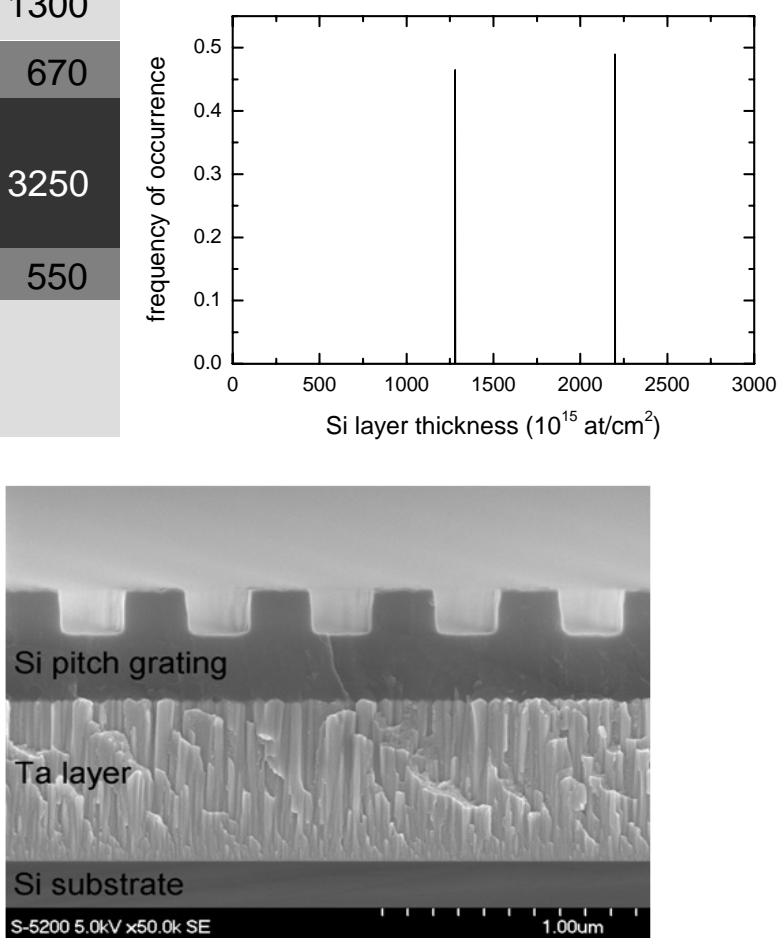

Fig. 3. Top: Measured and simulated RBS spectra from a $\mathrm{Si} / \mathrm{Ta} / \mathrm{Si}$ sample using two different sample models, which are shown in the middle. The measurement was made with $1.5 \mathrm{MeV}^{4} \mathrm{He}$ ions at normal incidence, scattering angle $165^{\circ}$. The outgoing beam was parallel to the grating, i.e. out from the visible plane. Simulations by SIMNRA 6.52 [42]. Model 1 assumes a layered sample structure, the layer compositions and thicknesses (in $10^{15}$ at. $/ \mathrm{cm}^{2}$ ) are indicated. Model 2 assumes a $3750 \times 10^{15}$ at. $/ \mathrm{cm}^{2}$ thick Ta layer. On top of the Ta layer a laterally inhomogeneous pure Si layer is deposited. The layer has of 2 different thicknesses, the frequency of occurrence of each thickness is indicated. Bottom: Scanning electron micrograph of the sample cross-section, from [64]. 